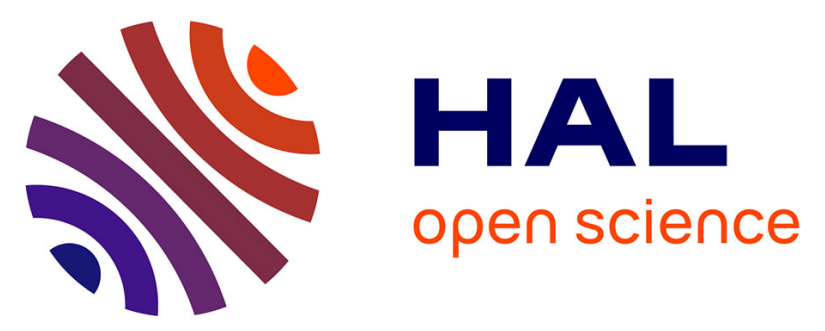

\title{
Simulating the detection and classification of high-redshift supernovae with HARMONI on the ELT
}

Sophie Bounissou, Niranjan Thatte, Simon Zieleniewski, Ryan C.W. Houghton, Mathias Tecza, Isobel Hook, Benoît Neichel, Thierry Fusco

\section{- To cite this version:}

Sophie Bounissou, Niranjan Thatte, Simon Zieleniewski, Ryan C.W. Houghton, Mathias Tecza, et al. Simulating the detection and classification of high-redshift supernovae with HARMONI on the ELT. Monthly Notices of the Royal Astronomical Society, 2018, 478 (3), pp.3189 - 3198. 10.1093/mnras/sty376 . hal-01936870

\section{HAL Id: hal-01936870 https://hal.science/hal-01936870}

Submitted on 3 Sep 2021

HAL is a multi-disciplinary open access archive for the deposit and dissemination of scientific research documents, whether they are published or not. The documents may come from teaching and research institutions in France or abroad, or from public or private research centers.
L'archive ouverte pluridisciplinaire HAL, est destinée au dépôt et à la diffusion de documents scientifiques de niveau recherche, publiés ou non, émanant des établissements d'enseignement et de recherche français ou étrangers, des laboratoires publics ou privés. 


\title{
Simulating the detection and classification of high-redshift supernovae with HARMONI on the ELT
}

\author{
S. Bounissou, ${ }^{1 \star}$ N. Thatte, ${ }^{2 \star}$ S. Zieleniewski, ${ }^{2}$ R. C. W. Houghton, ${ }^{2}$ M. Tecza, ${ }^{2}$ \\ I. Hook ${ }^{3}$ B. Neichel ${ }^{1}$ and T. Fusco ${ }^{1}$ \\ ${ }^{1}$ Aix Marseille University, CNRS LAM (Laboratoire d'Astrophysique de Marseille) UMR 7326, F-13388 Marseille, France \\ ${ }^{2}$ Department of Physics, University of Oxford, Denys Wilkinson Building, Keble Road, Oxford OX1 3RH, UK \\ ${ }^{3}$ Physics Department, Lancaster University, Lancaster, Lancashire LA1 4YB, UK
}

Accepted 2018 February 9. Received 2017 December 29; in original form 2017 May 5

\begin{abstract}
We present detailed simulations of integral field spectroscopic observations of a supernova in a host galaxy at $z \sim 3$, as observed by the HARMONI spectrograph on the Extremely Large Telescope, assisted by laser tomographic adaptive optics. The goal of the simulations, using the HSIM simulation tool, is to determine whether HARMONI can discern the supernova type from spectral features in the supernova spectrum. We find that in a $3 \mathrm{~h}$ observation, covering the near-infrared $H$ and $K$ bands, at a spectral resolving power of $\sim 3000$, and using the $20 \times 20$ mas spaxel scale, we can classify Type Ia supernovae and their redshift robustly up to $80 \mathrm{~d}$ past maximum light ( $20 \mathrm{~d}$ in the supernova rest frame). We show that HARMONI will provide spectra at $z \sim 3$, which are of comparable (or better) quality to the best spectra we can currently obtain at $z \sim 1$, thus allowing studies of cosmic expansion rates to be pushed to substantially higher redshifts.
\end{abstract}

Key words: instrumentation: adaptive optics - instrumentation: high angular resolutioninstrumentation: spectrographs - supernovae: general-galaxies: high-redshift.

\section{INTRODUCTION}

The use of Type Ia supernovae (SNe Ia) as cosmological distance indicators provided the first direct evidence for the accelerating expansion of the Universe (Riess et al. 1998; Perlmutter et al. 1999). Since then, developments in instrumentation and detector technology combined with major investments in telescope time from both ground- and space-based observatories have increased the number of distant SNe Ia useable for cosmology to several hundred, maintaining SNe Ia as a leading method for measuring the cosmological parameters. The next decade will see a further dramatic advance in the telescopes and instruments available for finding and studying SNe. In particular, the Large Synoptic Survey Telescope (LSST, Gressler 2016), the James Webb Space Telescope (JWST, Greenhouse 2016), the Euclid mission (Racca et al. 2016), the Wide Field InfraRed Survey Telescope (WFIRST, Goullioud et al. 2012), and Extremely Large Telescopes on the ground: the Giant Magellan Telescope (GMT, McCarthy et al. 2016), the Thirty Metre Telescope (TMT, Sanders 2013), and The European Extremely Large Telescope (E-ELT, Tamai et al. 2016) are all due to seeing the first light within the next decade. In this paper, we explore in detail

^ E-mail: sophie.bounissou@centrale-marseille.fr (SB); niranjan.thatte@ physics.ox.ac.uk (NT) the prospects for using the E-ELT with its first light spectrograph HARMONI (Thatte et al. 2014) for spectroscopy of SNe Ia.

\subsection{Supernova cosmology}

The discovery of the accelerating expansion of the Universe from measurements of distant SNe Ia (Riess et al. 1998; Perlmutter et al. 1999) has profound implications for cosmology. The cause of the acceleration is not understood and has led to the postulate that some form of 'Dark Energy', with an effective negative pressure, is driving the acceleration (Huterer \& Turner 1999). An alternative possibility is that the theory of General Relativity does not correctly describe gravity on very large scales (Dvali, Gabadadze \& Porrati 2000; Deffayet, Dvali \& Gabadadze 2002). Either of these possibilities would represent a fundamental change in our understanding of the Universe.

The quest to understand the acceleration of the Universe has motivated bigger and better (in terms of data quality) SN surveys. The results from the Supernova Legacy Survey (SNLS; Astier et al. 2006; Conley et al. 2011), combined with nearby SNe Ia samples and constraints from WMAP7 power spectrum (Larson et al. 2011), the Sloan Digital Sky Survey luminous red galaxy power spectrum (Reid et al. 2010), and constraints on the Hubble constant from SHOES (Riess et al. 2009, 2011), confirmed the accelerating expansion of the Universe and placed constraints on the dark 
energy equation of state parameter $(w=P / \rho)$ to within 7 per cent (including all statistical and SN Ia systematic errors), assuming a flat universe and a constant equation of state (Sullivan et al. 2011). These SNe combined with Planck CMB (Planck Collaboration I 2014) and baryon acoustic oscillations results (Beutler et al. 2011; Padmanabhan et al. 2012; Anderson et al. 2012) provide constraints on the parameters describing the accelerating Universe.

These measurements are consistent with the value of $w=-1$ expected if Dark Energy is equivalent to the cosmological constant. However, there is still room for a wide range of competing models (Rubin et al. 2009). A key test within reach of existing facilities is to determine whether or not $w$ varies with cosmic time. If any variation is found, this would immediately rule out the cosmological constant explanation for Dark Energy, with profound consequences for cosmology.

SN Ia studies are still the most efficient way to test for Dark Energy at high redshift and to measure its evolution with cosmic time. Currently, $w(z)$ is only well-constrained for redshifts $z<1$. Indeed, the commonly used parametrization of time-varying $w$, $w=w_{0}+w_{a} z /(1+z)$, leads to misleadingly tight constraints on the general form of $w$ at higher redshifts (Riess et al. 2007).

The next generation of facilities will see both a massive increase in statistics of SNe up to $z \sim 1$ and the possibility of studying $\mathrm{SNe}$ at very high redshifts up to $z \sim 4$ (Hook 2013). The Dark Energy Survey (DES) SN survey (Bernstein et al. 2012) is expected to find of order $3000 \mathrm{SNe}$ Ia, and LSST is expected to find hundreds of thousands over its 10 -yr survey lifetime out to $z \sim 0.8$ in its main survey, and further SNe to $z \sim 1$ in the Deep Drilling Fields (LSST Science Collaboration et al. 2009). This will enable fundamental tests, for example whether the measurement of $w$ is consistent in different directions on the sky. It will also enable detailed tests of the use of SNe Ia as distance indicators, for example by testing the results from SNe Ia with different host galaxy types.

To look for time variation in $w$ requires precision measurements over a significant baseline in cosmic time. This motivates the search for SNe Ia at redshifts significantly above $z=1$. Although SNe can be found up to $z \sim 1.5$ using 8 -m-class telescopes on the ground, the precision photometry required for cosmology requires observations that can only currently be achieved with the Hubble Space Telescope.

The ESA Euclid mission (Laureijs et al. 2011), which will have a wide-field near-infrared imaging capability, has the potential to observe SNe Ia out to $z \sim 1.6$ if it were to carry out a dedicated SN survey (Astier et al. 2014). The NASA-led WFIRST project is planning such a survey within its baseline mission, which will reach $z \sim 1.7 .{ }^{1}$ However, to reach even higher redshift requires new facilities with even higher sensitivity in the near-infrared, such as JWST and the ELTs. The increase in cosmic time baseline opens up the possibility of observing unexpected behaviour in the expansion history at early epochs.

\subsection{The importance of spectroscopy in SN Ia cosmology}

The use of SNe Ia for cosmology relies on spectroscopic redshift measurements of a subset of the sample to verify the photometric classification and secure the classification of the $\mathrm{SNe}$ as Type Ia.

\footnotetext{
${ }^{1}$ See the Final Report of the WFIRST-AFTA Science Definition Team: http://wfirst.gsfc.nasa.gov/science/sdt_public/WFIRST-AFTA_SDT_ Report_150310_Final.pdf
}

The redshift can be obtained from the $\mathrm{SN}$ itself or from its host galaxy (or both).

The SNe used in the Betoule et al. (2014) analysis were all classified spectroscopically, involving large investments of 8-m telescope time. For the much larger samples now being created by DES and in future by LSST, spectroscopy of every SN will not be possible. Instead, a combination of photometric classification of $\mathrm{SNe}$ and redshifts measured from host galaxies will be used. Although there has been great progress in recent years towards photometric classification of SNe, resulting in samples of SNe Ia with contamination as low as 4 per cent (Bazin et al. 2011; Campbell et al. 2013), a subset of SNe will always need to be spectroscopically classified in order to test the photometric classification.

For unambiguous classification of $\mathrm{SNe}$, high-quality spectroscopy of the SN itself is required (Filippenko 1997). Such SN spectra contain additional information that can be used to improve constraints yet further. First, since the details of SN spectra are sensitive to the details of the explosion, quantitative comparison of spectra as a function of redshift can provide constraints on the evolution of the SN population - an important test if SNe are to be used as 'standard candles' for cosmology (Hook et al. 2005; Maguire et al. 2012; Milne et al. 2015).

Secondly, SN spectra allow tests for variations in $\mathrm{SN}$ spectral properties with their host galaxy properties (Pan et al. 2015; Nordin et al. 2011). Thirdly, they allow tests for spectral indicators that correlate with SN luminosity and hence have the potential to reduce the scatter in the Hubble diagram (Bronder et al. 2008; Bailey et al. 2009; Blondin, Mandel \& Kirshner 2011; Foley \& Kasen 2011; Walker et al. 2011; Folatelli et al. 2013). All these spectral tests ultimately have the potential to improve SN Ia distance measurements and hence the constraints on cosmological parameters.

\subsection{Reaching higher redshift: E-ELT and HARMONI}

Currently, the most distant spectroscopically confirmed SN Ia is a gravitationally lensed SN at $z=1.35$ (Rodney et al. 2015). To reach higher redshifts for the general population of (unlensed) SNe Ia requires increased sensitivity.

Since SNe are point sources, their observation from the ground greatly benefits from adaptive optics correction. Below we demonstrate how the E-ELT's HARMONI spectrograph, assisted by laser tomographic adaptive optics (LTAO), could be used to observe and classify very distant SNe. Such SNe could possibly be found by JWST using deep, repeat imaging with NIRCAM. In principle, the E-ELT itself, equipped with its first light AO-fed imaging camera MICADO (Davies et al. 2016), would also have the sensitivity to find very distant SNe out to $z \sim 4$, although JWST NIRCAM is better suited to a high-redshift SN survey because of its wider field of view.

This paper is organized as follows: In Section 2, we describe the HARMONI simulator HSIM, which is used to carry out the simulations of expected performance. Section 3 explains how the input data cube is created. The actual simulation runs and the extraction of the spectra are explained in Section 4. Section 5 provides details of the analysis, and Section 6 summarizes the key conclusions of this work.

\section{THE HARMONI INSTRUMENT AND SIMULAT OR}

HARMONI will provide the first-light spectroscopic capability of E-ELT, which is due for completion in 2024 (Thatte et al. 2014). It 
will have a wavelength coverage from 0.5 to $2.45 \mu \mathrm{m}$ and will be able to take full advantage of both the E-ELT's large collecting area and angular resolution to achieve a wide range of scientific goals. Four spaxel scale configurations have been chosen: $4 \times 4,10 \times 10$, $20 \times 20$, and $30 \times 60$ mas, and three resolving powers $R(=\lambda / \Delta \lambda)$ of 3000,7000 , and 20000 . HARMONI will work in conjunction with two adaptive optics systems: LTAO [high-order correction using laser guide stars (LGS) over most of the sky], Single Conjugate Adaptive Optics [high-order correction using natural guide stars (NGS) in the vicinity of the bright reference star], or with no AO at all.

As the design phase is still ongoing, instrument simulations are vital to understanding the capabilities and limitations of the instrument design in achieving certain science goals. The HARMONI Simulator HSIM (Zieleniewski et al. 2015, source code: https://github.com/szieleniewski/HSIM) is a dedicated pipeline for simulating observations with HARMONI. HSIM is a powerful tool for quantifying the predicted HARMONI performance to assess the feasibility of science programmes. Specifically, HSIM takes an input 3D data cube $(x, y, \lambda)$, which contains the source flux, and then simulates an observation by adding sky, telescope, instrument, and detector effects. In this process, several parameters can be chosen for the simulated observations according to the science program. The result is a mock-observed data cube and by comparing it with the input data cube, users can determine how well the information would be recovered when observing with E-ELT and HARMONI. Hence, HSIM can predict HARMONI performance for specific science programmes and provides the user with important information on the observability of a source, therefore helping in the optimization of future science programmes for HARMONI on the E-ELT.

\section{SUPERNOVA IA SIMULATIONS INSIDE A GA L A X Y}

The main goal of this project is to simulate SNe Ia inside a galaxy at $z=3$ for different luminosities, which approximates different points on the light curve of the SN, and to determine what the E-ELT would be able to detect. This will yield a limiting magnitude, i.e. the magnitude beyond which the SN cannot be detected.

In this study, the input galaxy data cube is taken from the output of a large-scale cosmological simulation. We use a galaxy at $z=3$ from the RAMSES simulations (Teyssier 2002). The particular galaxy is extracted from the $z=3$ output of the NutFB simulation, described in detail by Powell, Slyz \& Devriendt (2011) and Kimm et al. (2011). The Nut suite is a collection of high-resolution cosmological simulations of a Milky Way-like galaxy forming at the intersection of three filaments in a $\Lambda$ cold dark matter cosmology. It has star formation rate (SFR) of $4 \mathrm{M}_{\odot} \mathrm{yr}^{-1}$, a luminosity of $0.3 \mathrm{~L}_{*}$ at this redshift (Ilbert et al. 2013), and thus represents an object where an SN might typically be found at this redshift. This galaxy was chosen because of the availability of high spatial resolution (12 pc) simulations of the starlight.

The simulations yield stellar population data from which star particles have been converted into stellar light. The input data cube $(x$, $y, \lambda)$ has a size of $(320,320,4000)$ and contains a 10-kpc galaxy (Fig. 1) as observed at $z=3$. It covers the $1.44-2.88 \mu \mathrm{m}$ wavelength range and the spectral sampling is $3.6 \AA$, whereas the spectral resolution is equal to $10 \AA$. Regarding the spatial dimension, the data cube has been sampled on a $4 \times 4$ mas grid of spaxels. Therefore, the mock galaxy is a data cube of size $1.28 \times 1.28 \operatorname{arcsec}^{2}$. Fig. 2 plots the spatially integrated spectrum of the galaxy at $z=3$. Note that the galaxy simulations have been focused on stellar absorption

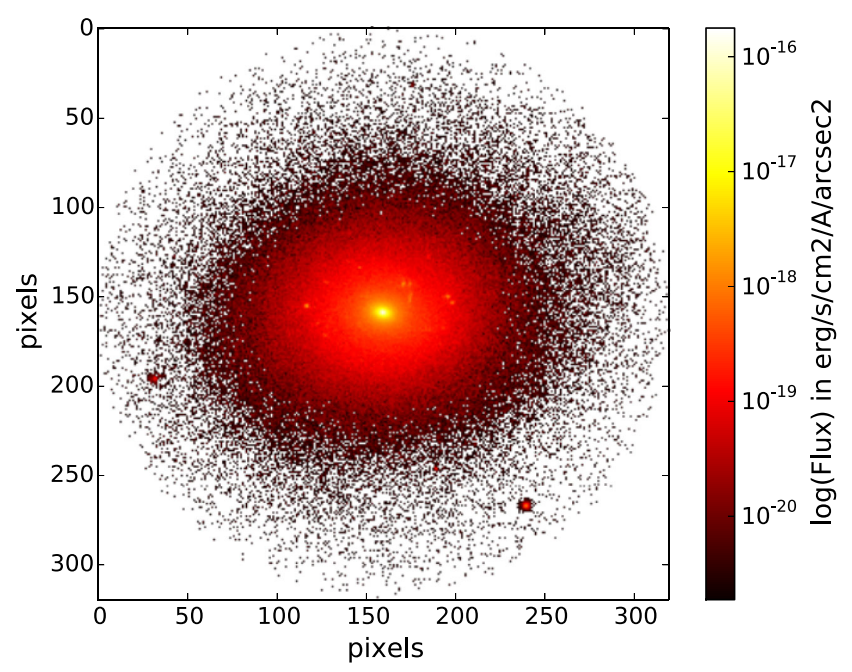

Figure 1. Median image of the galaxy data cube at redshift $z=3$. The spatial extent is $1.28 \times 1.28 \operatorname{arcsec}^{2}$ with 4 mas sampling. Note that the galaxy simulations have been focused on stellar light.

lines (for reasons independent of the present study), leading to a spectrum with no emission lines. The near-infrared $H$ and $K$ bands are shaded in orange and red in Fig. 2. Both will be used as filters for the SN observations.

\subsection{Magnitudes for the host galaxy}

Kendrew et al. (2016) compute the $V$-band absolute magnitude of the $N u t F B$ galaxy at -21.4 . Including the $K$-correction for magnitude calculations, described in Hogg et al. (2002), we obtain the $z=3$ apparent Vega magnitude of the galaxy as +23.3 in the $H$ band and +22.4 in the $K$ band. Integrated spectrum and magnitude calculations have been computed for an aperture size of $10 \mathrm{kpc}$ diameter. At $z=3$, the galaxy is at an angular size distance of $1613.6 \mathrm{Mpc}$, which gives a scale of $0.127 \mathrm{arcsec} \mathrm{kpc}{ }^{-1}$ and thus occupies a total $1.27 \mathrm{arcsec}$ field. The galaxy has a half-light radius of $0.3 \mathrm{kpc}$, or 40 mas, and a truncation radius of $1.5 \mathrm{kpc}$, or 0.2 arcsec.

\subsection{Computing SN magnitudes}

In the context of this study, relatively local $\mathrm{SNe}$ will be redshifted at $z=3$ by scaling their spectra. Richardson et al. (2014) presents the absolute-magnitude distribution of seven SN types (Table 1). Spectra for Types Ia, Ib/c, Ic and II are shown in Fig. 3 for the $350-600 \mathrm{~nm}$ range ( $B V$ band). These $\mathrm{SNe}$ have been observed in the nearby Universe at maximum light: Ia corresponds to SN1981B (Branch et al. 1983), Ib/c to SN1999EX (Hamuy et al. 2002), Ic to SN1994I (Filippenko et al. 1995), and II to SN1987A (Menzies et al. 1987).

For an $\mathrm{SN}$ observed at a redshift $z$, the spectrum is stretched by a factor of $(1+z)$, and so the spectral sampling (and resolution) will be multiplied by $(1+z)$. The redshifted flux can be calculated with the following equation:

$F_{\lambda, z}=F_{\lambda, z=0}\left(\frac{10 \mathrm{pc}}{d_{\mathrm{L}}}\right)^{2}(1+z)^{-1}$,

where $z$ is the redshift, $F_{\lambda, z=0}$ and $F_{\lambda, z}$ are the rest-frame and redshifted fluxes, respectively, and $d_{\mathrm{L}}$ is the luminosity distance. The 


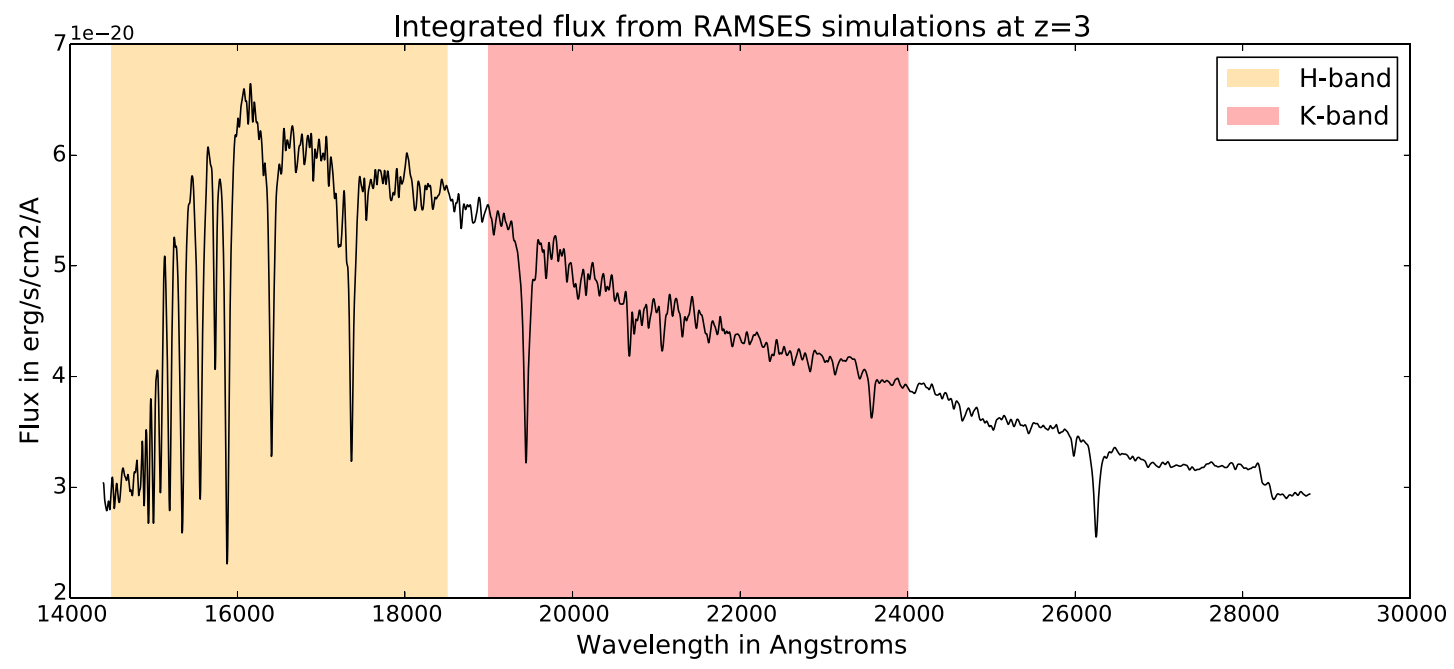

Figure 2. Integrated stellar spectra of the $z=3$ RAMSES galaxy as observed (redshifted) between 1.44 and $2.88 \mu \mathrm{m}$. Note that the galaxy simulations have been focused on stellar absorption lines (no emission lines). The near-infrared $H$ and $K$ bands have been shaded in orange and red, respectively.

Table 1. Absolute magnitude of seven SN types. As expected, Type Ia is the brightest event. Reproduced from Richardson et al. (2014).

\begin{tabular}{lcc}
\hline SN type & Absolute $B$ magnitude & Standard deviation \\
\hline Ia & $-19.25 \pm 0.20$ & 0.50 \\
Ib & $-17.45 \pm 0.33$ & 1.12 \\
Ic & $-17.66 \pm 0.40$ & 1.18 \\
IIb & $-16.99 \pm 0.45$ & 0.92 \\
IIL & $-17.98 \pm 0.34$ & 0.86 \\
IIP & $-16.75 \pm 0.37$ & 0.98 \\
IIn & $-18.53 \pm 0.32$ & 1.36 \\
\hline
\end{tabular}

$(1+z)$ factor is due to the bandpass stretching. To derive the luminosity distance at $z=3$, we use the Ned Wright's Javascript Cosmology Calculator (Wright 2006) with cosmological parameters as follows: $\Omega_{\mathrm{M}}=0.258, \Omega_{\Lambda}=0.742$, and $H_{0}=72 \mathrm{~km} \mathrm{~s}^{-1} \mathrm{Mpc}^{-1}$, yielding $d_{\mathrm{L}}=25817.9 \mathrm{Mpc}$.

At $z=3$, the observed apparent magnitude of the SNe can be computed using the rest-frame $B$-band absolute magnitudes listed in Table 1 and then redshifting using equation (1). In detail, the

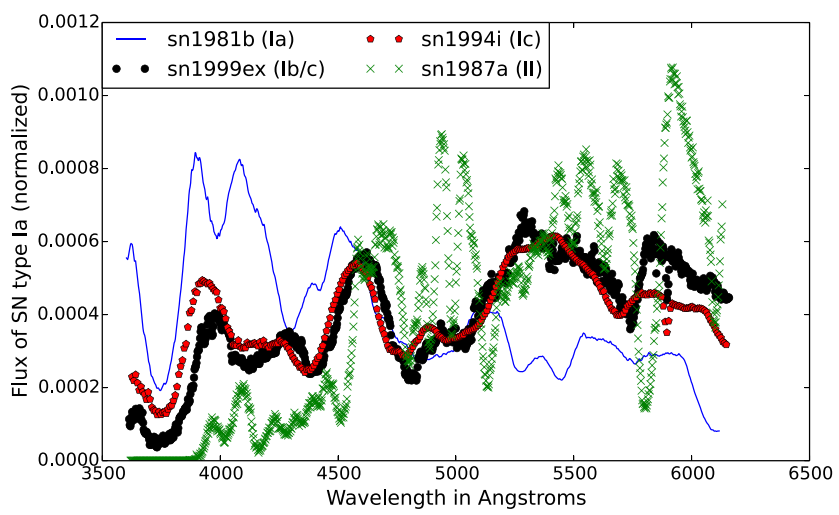

Figure 3. Spectra $\left(\mathrm{F}_{\lambda}\right)$ of Types Ia, Ib/c, Ic, and II over the BV range. Spectra have been normalized to compare features. Note that for one spectrum the distinction between Ib and Ic is quite blurred. Spectra data are extracted from the SUPERFIT library (Howell et al. 2005, source code: http://www.dahowell.com/superfit.html)
Table 2. Relationship between luminosity of the $\mathrm{SN}$ at maximum light and luminosity of the galaxy, in $H$ band. To calculate the Type $\mathrm{Ib} / \mathrm{c}$ ratio, we used Type $\mathrm{Ib}$ absolute magnitude, and to compute the Type II ratio, we used Type IIL absolute magnitude.

\begin{tabular}{lc}
\hline SN type & Ratio \\
\hline $\mathrm{Ia}$ & $0.243 \pm 0.045$ \\
$\mathrm{Ib}(/ \mathrm{c})$ & $0.039 \pm 0.012$ \\
$\mathrm{Ic}$ & $0.050 \pm 0.019$ \\
$\mathrm{II}$ & $0.038 \pm 0.012$ \\
\hline
\end{tabular}

known $B$-band magnitude is used to determine the flux scaling for the spectrum. The observed, redshifted spectrum, computed using equation (1), is then used to determine the observed $H$-band magnitude of the SN, which is compared with the integrated $H$-band light of the host galaxy. Thus, we get the ratio between luminosity of the $\mathrm{SN}$ and luminosity of the galaxy in $H$ band (reported in Table 2). At maximum light, the ratio between $\mathrm{SN}$ and galaxy brightness is 0.24 . We use the integrated galaxy light for comparison, as the $\mathrm{SN}$ is likely to be identified using ground-based surveys only if it causes a significant increase in the overall observed magnitude of the galaxy (approx $\geq 0.1 \mathrm{mag}$ ).

This study has been performed with SN Ia simulations because it is considered as a standard candle and, for equal redshift, Type Ia is brighter than the core-collapse SNe, but simulations of Type Ib, Ic, or II could be computed similarly. A spectrum showing Si II features in Type Ia has been plotted in Fig. 4, as spectroscopy is the most reliable diagnostic to classify different $\mathrm{SNe}$. This spectrum has been integrated into the input data cube of the galaxy (Fig. 1) at a position corresponding to a point source (single spaxel) at 0.2 arcsec from the galaxy centre. The distance was chosen to correspond to the truncation radius of the galaxy, or five times the effective radius. We realize that this value is close to the largest separation expected. However, we chose to bias our study in this way, as the E-ELT will not be used to detect the $\mathrm{SNe}$, and most ground-based surveys would struggle to identify $\mathrm{SNe}$ close to the galaxy nucleus. A full study covering a range of different distances and redshifts is beyond the scope of this paper. 


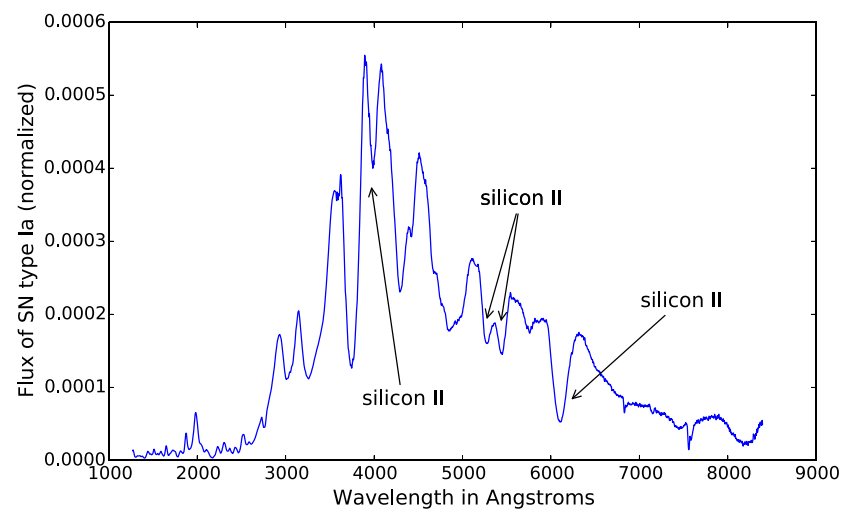

Figure 4. Normalized spectrum of an SN Ia (SN1981B) at maximum light over the $\sim 1200-8500 \AA$ wavelength range. $\mathrm{Si}$ II absorption features are represented by arrows. This spectrum is extracted from the suPERFIT library (Howell et al. 2005, source code: http://www.dahowell.com/superfit.html)

\section{SIMULATING SN IA OBSERVATIONS USING HSIM}

The purpose of the simulations is to simulate the fading of the $\mathrm{SN}$ brightness over time, starting at the peak of the light curve. Assuming that the $\mathrm{SN}$ is at maximum light on the light-curve yields a ratio between luminosity of the $\mathrm{SN}$ and the galaxy of 0.24 . Starting at this value, we produce several data cubes containing a mockobserved SN Ia at different luminosities. In practice, the chosen ratios are $0.24,0.075,0.05,0.035,0.024$, and 0.01 .

One can roughly estimate a date corresponding to the days following the maximum light of the light curve of Perlmutter (2003), assuming the shape of the light curve is the same at $z=3$ as in the nearby Universe. For instance, if the ratio of 0.24 is the maximum on the light curve, a ratio of 0.024 is 10 times fainter, leading to an addition of 2.5 in magnitude so we are $\sim 20 \mathrm{~d}$ after the maximum light in the rest frame. As the $(1+z)$ factor stretches spectra, it also stretches time, meaning that a ratio of 0.024 corresponds to $80 \mathrm{~d}$ in observed frame. Many points corresponding to the ratios mentioned above were studied between the maximum and $80 \mathrm{~d}$ after it, i.e. up to a ratio of 0.024 , where the $\mathrm{SN}$ is almost undetectable. In this study, only one spectrum template is used to simulate the $\mathrm{SN}$ at different epochs, so we have ignored the fact that the spectrum evolves as the SN fades.

\subsection{Running HSIM}

HSIM has been run to simulate observations and produce mock observed data cubes. Parameters of the observations have been listed in Table 3. Three-hour exposure time $(12 \times 900 \mathrm{~s}$ to avoid detector saturation) mock observations are simulated with an output spaxel scale of $20 \times 20$ mas. The choice of this scale has been justified in the literature: Zieleniewski et al. (2015) has shown that the $20 \times 20$ mas scale provides the best sensitivity among HARMONI spaxel scales for point sources. We have deliberately chosen to ignore the convolution of the wavelength dimension with the instrumental width because the input data cube has a coarser resolution $(\Delta \lambda=$ $10 \AA$ ) than the lowest resolution gratings in HARMONI (for $H / K$ bands $\Delta \lambda=19500 / 3000 \sim 6.5 \AA$ ), so the simulator maintains the input spectral resolution. Lastly, we use point spread functions (PSFs) simulating LTAO with six LGS to correct distortion from the atmosphere towards the galaxy. The PSF for each wavelength in this LTAO data cube is the result of a nine layers atmospheric
Table 3. HSIM input parameters for observing the SN Ia with HARMONI. Note that the spectral dimension has been ignored because the input resolution is coarser than the outputs obtained with HARMONI spectrograph.

\begin{tabular}{lc}
\hline HARMONI simulator input parameters \\
\hline DIT & $900 \mathrm{~s}$ \\
NDIT & 12 \\
$X$ scale & $20 \mathrm{mas}$ \\
$Y$ scale & $20 \mathrm{mas}$ \\
Grating & $H+K$ \\
AO & LTAO \\
\hline
\end{tabular}

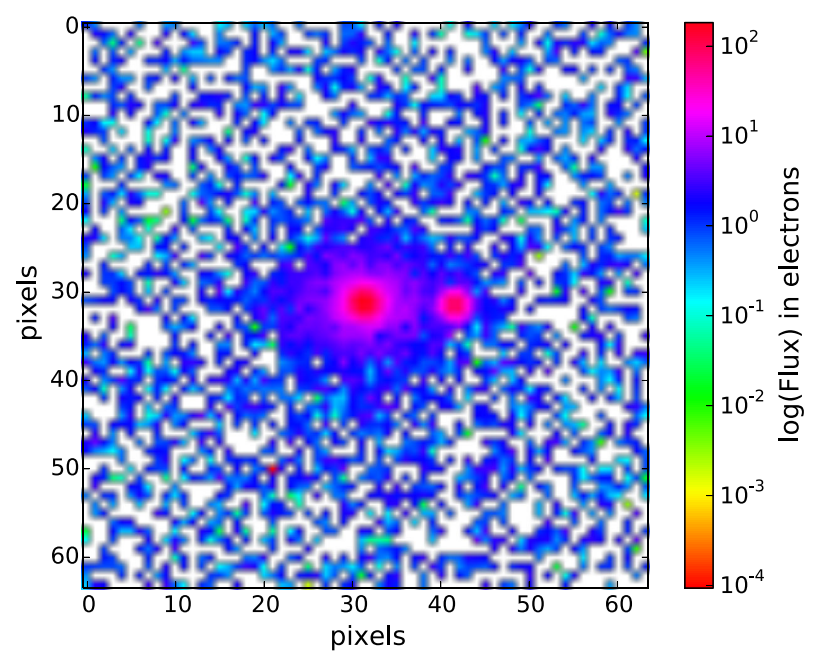

Figure 5. View of the observed data cube at $20 \times 20$ mas with the $\mathrm{SN}$ within the host galaxy at maximum light, obtained by calculating the median for each spaxel of the sky-subtracted data cube. The SN Ia has been simulated at 0.2 arcsec on the right of the centre of the galaxy, namely 10 spaxels.

reconstruction and the seeing was fixed at 0.64 arcsec, which means optimistic reconstruction and median seeing conditions (T. Fusco, private communication).

For all simulations, HSIM returns a mock observed data cube and a background cube (among others). Fig. 5 shows the observed SN within the host galaxy at maximum light $\sim 10$ spaxels to the right of the centre of the galaxy. The same view of resulting data cube at different times after maximum light is shown in Appendix A1. Output cubes have a size of $(64,64,2805)$ with a spatial sampling of $20 \times 20$ mas and $3.6 \AA$ spectral sampling (same as the input data cube). Furthermore, observation with no SN, which would correspond to an observation one year later for instance, has also been simulated in the same observing conditions (i.e. with the same PSF data cube).

\subsection{Optimizing the signal-to-noise ratio}

Once the observation is simulated through HSIM, one needs to extract the spectrum of the observed SN. In order to do this, one must choose the size of the aperture, which will be used to extract this spectrum. The criterion for this is the signal-to-noise ratio $(\mathrm{S} / \mathrm{N})$; indeed, the extraction aperture size will be the one that optimizes the $\mathrm{S} / \mathrm{N}$.

In practice, among the different output data cubes from HSIM, one gets the variance of the reduced cube (background subtracted observed cube). This cube corresponds to the square of the 


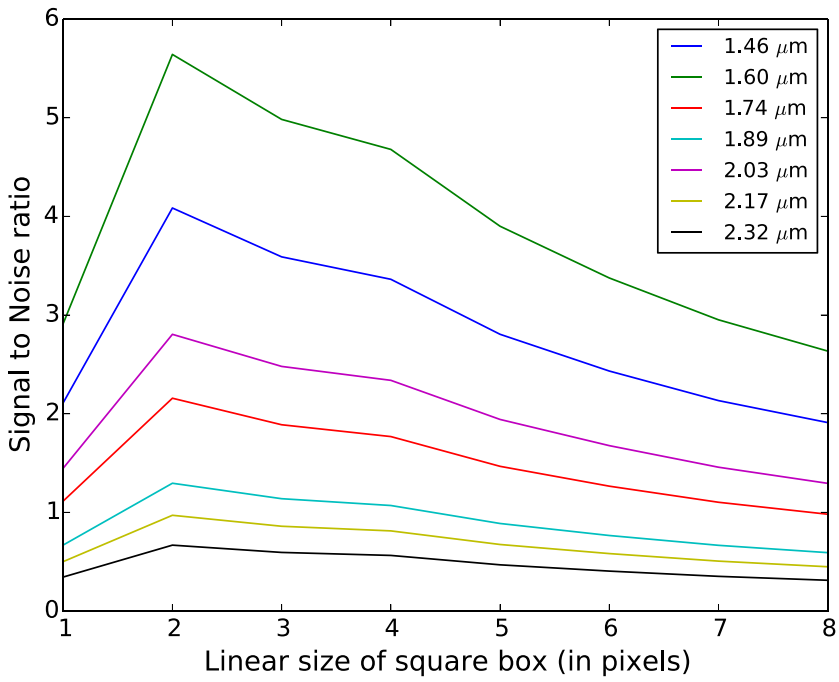

Figure 6. SNR at maximum light for several aperture sizes (in pixels) centred on the SN location. Calculations have been performed for all wavelengths between 1.45 and $2.45 \mu \mathrm{m}$, a few representative wavelengths are shown in the plots. To avoid galaxy contamination, an observation of the galaxy one year later has been subtracted from the observation including the $\mathrm{SN}$. Note that this operation adds noise.

standard deviation in the $\mathrm{S} / \mathrm{N}$ formula. The mean signal is given by the noiseless object data cube (also provided by HSIM) and the combination of this with the statistical variance provides an estimate of the S/N. Fig. 6 plots the $\mathrm{S} / \mathrm{N}$ at maximum light for several aperture sizes centred on the SN location (nominal position from input cube). Calculations have been carried out for different wavelengths between 1.45 and $2.45 \mu \mathrm{m}$. To avoid galaxy contamination, the galaxy data cube without SN (simulating an observation made one year later with the same parameters) has been subtracted from the observations with the SN, which adds noise to the SN observation and thus adds a variance term when calculating the $\mathrm{S} / \mathrm{N}$. The figure suggests that extracting the $\mathrm{SN}$ in a $2 \times 2$ spaxel box, namely a $40 \times 40$ mas box, yields the best $\mathrm{S} / \mathrm{N}$, independent of wavelength. Note that there is no general trend to increase or decrease the $\mathrm{S} / \mathrm{N}$ plot as a function of wavelength, this may be due to the emission and absorption lines of the sky, which result in enhanced photon noise at specific wavelengths, or due to broad spectral features in the $\mathrm{SN}$ spectrum. The shape of the $\mathrm{S} / \mathrm{N}$ curve can be explained by the fact that at the beginning an increase of the box size gathers more signal from the object but the noise becomes more important once we increase past a specific size (here $2 \times 2$ spaxels).

To increase $\mathrm{S} / \mathrm{N}$, particularly for fainter $\mathrm{SNe}$, we rebin the spectral dimension. This can be done without loss of information, as most spectral features in the SN spectra are broad. In this study, the input data has a coarser resolution $(\Delta \lambda \sim 10 \AA)$ than the HARMONI resolution. Hence, we can bin the spectral dimension, care being taken not to degrade the resolution too much because we want to be able to detect spectral features. For this, we choose to bin the maximum light spectrum by 9 pixels, so that the mean $\mathrm{S} / \mathrm{N}$ of the maximum light is multiplied by $\sim 3$ (Fig. 7). As the luminosity of the SN decreases, we have increased the binning so as to achieve a robust cross-correlation signal. The chosen binning in pixels and in $\AA$, and the resulting mean S/N computed over the entire $H$ and $K$ bands is listed in Table 4.

Therefore, to have exploitable data, we have optimized the aperture size and the binning to increase the $\mathrm{S} / \mathrm{N}$ of spectra. We have not corrected for the narrow telluric absorption features in the simulated

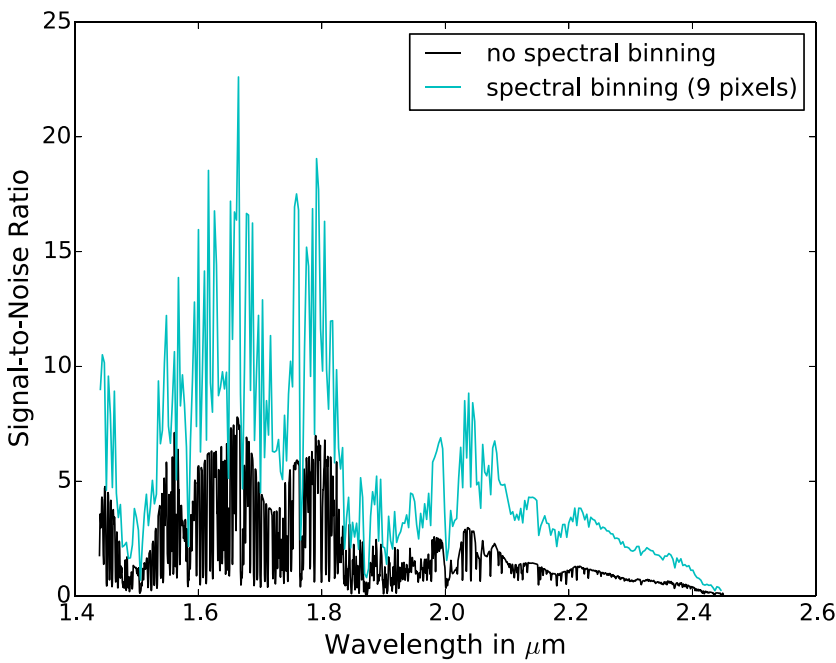

Figure 7. $\mathrm{S} / \mathrm{N}$ at maximum light for the wavelength range $1.45-2.45 \mu \mathrm{m}$ in $3 \mathrm{~h}$. The black curve is the result of a $10 \AA$ spectral resolution, whereas for the green one we have degraded the spectral resolution by applying a 9 pixel boxcar smoothing to increase the $\mathrm{S} / \mathrm{N}$ and give more confidence to the output signal. The galaxy has not been subtracted.

Table 4. Binning (in pixels and $\AA$ ) and resulting averaged $\mathrm{S} / \mathrm{N}$ for different SN luminosity. Trade-offs have been made between spectral resolution and $\mathrm{S} / \mathrm{N}$.

\begin{tabular}{lccc}
\hline $\begin{array}{l}\text { Ratio } \\
\text { SN/galaxy }\end{array}$ & $\begin{array}{c}\text { Spectral } \\
\text { binning (pixels) }\end{array}$ & $\begin{array}{c}\text { Spectral } \\
\text { bin }(\AA)\end{array}$ & $\begin{array}{c}\text { Mean S/N } \\
\text { (binned) }\end{array}$ \\
\hline 0.24 & 9 & 32.4 & 5.38 \\
0.075 & 16 & 57.6 & 2.27 \\
0.050 & 25 & 90 & 1.89 \\
0.035 & 36 & 129.6 & 1.61 \\
0.024 & 64 & 230.1 & 1.48 \\
0.010 & 81 & 291.6 & 0.68 \\
\hline
\end{tabular}

observed spectra, but have excluded the region between the $H$ and $K$ bands, where telluric absorption is strong. In the next section, spectra are extracted to get the most reliable information and to classify the SN as Type Ia. Note that the same process could be conducted to analyse any of the SNe types.

\section{SUPERNOVA CLASSIFICATION}

SN classification can be reliably made from a study of the spectrum. Light curves can help, but only if the flux at maximum light can be reliably estimated, and the redshift is known independently (e.g. using the host galaxy). For high redshift SN such as those analysed here, lack of spatial resolution will be a strong limitation in reliable light curve measurements. Hence, spectral analysis stands as the most reliable tool for classification. In the following, we choose to focus on two methods for analysis of spectra for classification: the cross-correlation and the $\chi^{2}$ methods.

\subsection{Cross-correlation method}

Once the aperture size is chosen so as to optimize the $\mathrm{S} / \mathrm{N}$, we can extract the spectrum of the SN for data processing. Fig. 8 displays the extracted spectrum and the associated background variance of the SN at maximum light. Note that the flux has been converted from electron counts to energy in order to be consistent with the 


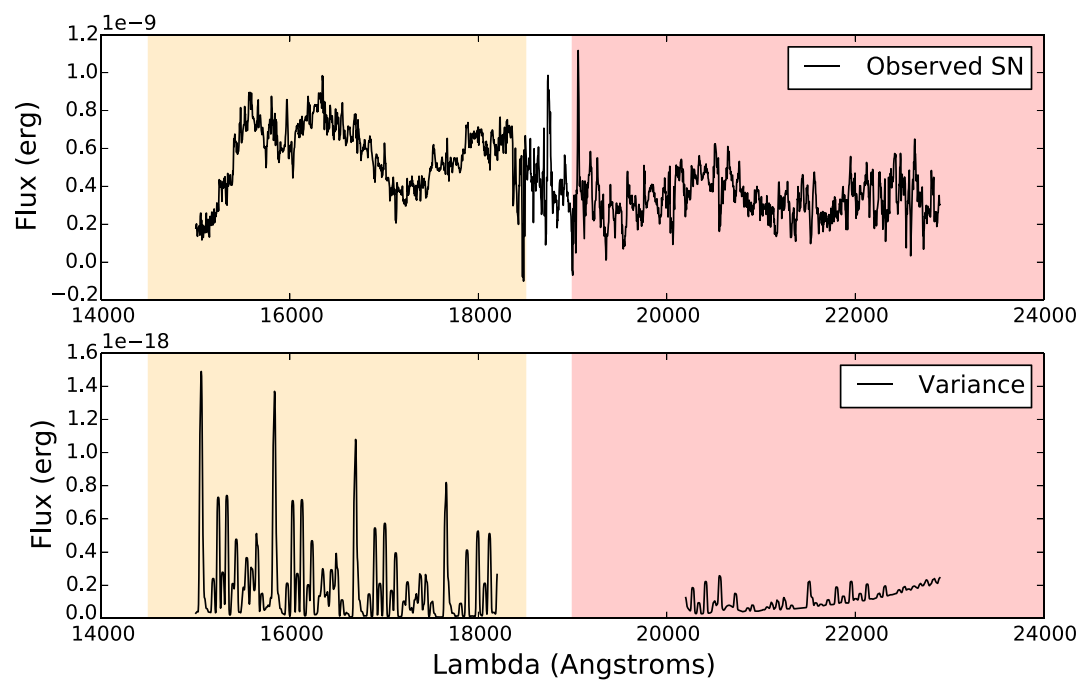

Figure 8. Extracted spectrum and associated background variance at maximum light with a rebinning of 9 pixels. The $H$ and $K$ bands are shaded in orange and red, respectively. Note that the flux has been converted from electrons counts to energy in order to be consistent with the initial SN templates, and that the flux has been divided by the transmission curve, which leads to increase the variance between the $H$ and $K$ bands. The galaxy flux has not been subtracted.

initial SN templates, and that the flux has been divided by the transmission curve, which leads to increase the variance between the $H$ and $K$ bands. As previously mentioned, the extraction aperture was a $40 \times 40$ mas box. In the case of maximum light, the spectral flux has been calculated by first doing a median in a nine spectral pixels running boxcar in order to remove photon noise from bright sky lines and then averaging over the four spatial pixels of the $\mathrm{SN}$. The variance is the result of the background variance (main source of photon noise), and it has been computed by first taking the average of the nine spectral pixels and then averaging over the four spatial pixels. A mean-to-median correction factor multiplies the spectrally averaged variance to use the same statistics over the input signal and the variance. The same process is applied for each SN luminosity following the previously chosen rebinning (Table 4). In both calculations, specific attention has been paid to take into account the photon noise impact. However, note that in this way although we get smoother observed flux for brighter $\mathrm{SN}$, we also lose key features within fainter SN when rebinning.

The previous calculations are useful for computing the crosscorrelation factor. The cross-correlation is used in signal processing as a measure of the similarity of two signals as a function of the lag of one relative to the other. Here, we assume there is no lag between the spectra we will compare; in other words, we assume the redshift is perfectly known; this may be done using galaxy data. As a reminder, the main goal of this study is only to know if $\mathrm{SNe}$ can be observed at high redshift, namely $z=3$, with HARMONI. In short, we measure the similarity of the observed spectrum at high $z$ and those already observed in the nearby Universe, to retrieve the $\mathrm{SN}$ type. This will be done with a weighted cross-correlation factor $\Gamma$ expressed as follows:

$\Gamma=\frac{\sum_{i=1}^{n} w_{i}\left(x_{i}-x_{0}\right)\left(y_{i}-y_{0}\right)}{\sqrt{\sum_{i=1}^{n} w_{i}\left(x_{i}-x_{0}\right)^{2} \sum_{i=1}^{n} w_{i}\left(y_{i}-y_{0}\right)^{2}}}$,

where $\left(x_{i}\right)_{i \in N}$ and $\left(y_{i}\right)_{i \in N}$ are two series, $x_{0}$ and $y_{0}$ are their respective means, and $w_{i}$ is the weighting function corresponding here to the inverse of the variance and thus reducing the impact of the photon noise in the $\Gamma$ calculation.
Table 5. Cross-correlation factor $\Gamma$ between observed Ia and other spectra at different flux levels w.r.t the host galaxy (corresponding to different times past maximum light). Ia* corresponds to SN1981B, Ia corresponds to SN2002BO, Ib corresponds to SN1999EX, Ic corresponds to SN1994I, and II corresponds to SN1987A. Ia* is the input spectrum used for simulations. Calculations have been made over the wavelength ranges 1.5-1.82 and 2.02$2.29 \mu \mathrm{m}$. Negative values for $\Gamma$ mean the two spectra are anti-correlated, whereas values close to zero mean there is no correlation.

\begin{tabular}{llcrrr}
\hline & Ia $*$ & Ia & Ib & Ic & \multicolumn{1}{c}{ II } \\
\hline 0.24 & 0.761 & 0.590 & -0.495 & -0.387 & -0.606 \\
0.075 & 0.622 & 0.462 & -0.459 & -0.352 & -0.517 \\
0.050 & 0.663 & 0.503 & -0.482 & -0.447 & -0.550 \\
0.035 & 0.504 & 0.375 & -0.366 & -0.278 & -0.384 \\
0.024 & 0.421 & 0.255 & -0.274 & -0.216 & -0.343 \\
0.010 & -0.159 & -0.158 & 0.144 & 0.150 & 0.191 \\
\hline
\end{tabular}

In Table 5, we have compared the observed $\mathrm{SN}$ at different times past maximum light and with different spectra, namely Ia, Ib, Ic, and II. The notation 'Ia*' means this is the input spectrum used for simulations. To avoid biasing the simulation, we have set all SN types to the same peak absolute luminosity. We introduce another spectrum (marked 'Ia') that has (also) been extracted from SUPERFIT library (http://www.dahowell.com/superfit.html) and corresponds to the SN SN2002BO (Benetti et al. 2004) one day before maximum light. Calculations have been made in the $H+K$ band, but in order to remove effects due to low atmospheric transmission between 1.82 and $2.02 \mu \mathrm{m}$, cross-correlation is calculated over two wavelength ranges $1.5-1.82$ and $2.02-2.29 \mu \mathrm{m}$. Negative values for $\Gamma$ mean the two spectra evolve in opposite ways, whereas values close to zero mean there is no correlation. The cross-correlation yields the highest value for both Type Ia (note that each time the best result is obtained for the spectrum used to simulate the $\mathrm{SN}$ ) and is far superior to the others types up to $r=0.024$. We have carried out calculations for $r=0.01$ to show that the signal is too low to recover any information, indeed even with a binning of 81 spectral pixels, the mean $\mathrm{S} / \mathrm{N}$ is too small $(\sim 0.7)$ and the cross-correlation calculations are not relevant at all. From values of the ratio fainter than $r=0.024$, the SN type is undetectable and extracting a spectrum mismatches the SN type. 


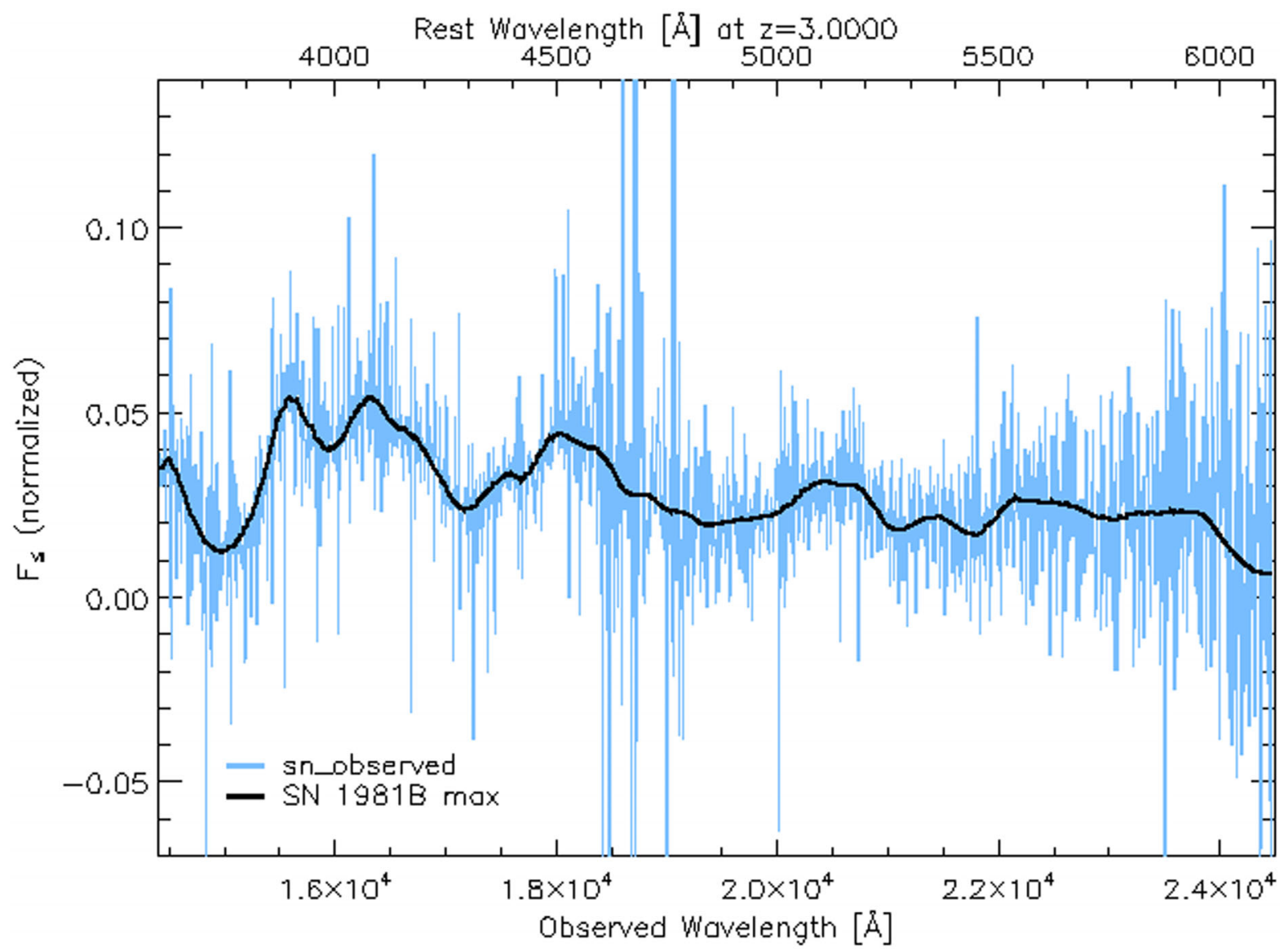

Figure 9. SUPERFIT best match at maximum light. In blue, the observed spectrum (minus the galaxy spectrum) and in black, the best match spectrum. In this particular case, the best match is obtained for the spectrum used for simulations. SUPERFIT gives the value of the optimized parameters, such as the best-fitting redshift $z$. At maximum light, the best fitting is obtained for $z=3.000 \pm 0.005$. Note that the increased noise around $1.9 \mu \mathrm{m}$ is due to the poor transmission between the atmospheric windows $H$ and $K$.

\subsection{Minimum $\chi^{2}$ method}

The $\chi^{2}$ method is the fitting method used in SUPERFIT by Howell et al. (2005) to find the best match to an observed spectrum from a library of hundreds of previously observed spectra of SNe. SUPERFIT computes $\chi^{2}$ to try to minimize it and thus recover the SN type. $\chi^{2}$ depends on three parameters: the redshift $z$, the amount of galaxy contamination, and the reddening law $A_{\lambda}$. As a consequence, $\chi^{2}$ is calculated as follows:

$\chi^{2}=\sum_{\lambda} \frac{\left[O(\lambda)-(\mathrm{cc}) \times T(\lambda, z) 10^{c A_{\lambda}}-(\mathrm{ff}) \times G(\lambda, z)\right]^{2}}{\sigma^{2}(\lambda)}$,

where $\chi^{2}$ is calculated for spectra at a given redshift $z ; O$ is the observed SN spectrum, $T$ is an $\mathrm{SN}$ spectrum template to which the observed $\mathrm{SN}$ is compared, $G$ is the observed galaxy spectrum, and $\sigma^{2}$ is the variance associated with the observation. Constants $\mathrm{cc}, \mathrm{ff}$, and $\mathrm{c}$ are optimized to minimize $\chi^{2}$.

The SUPERFIT output is a list of matches depending on the goodness of fit to the observed SN spectrum. The SUPERFIT program has been tested on the simulated spectra of this study. In the same way than in the cross-correlation method, spectra of the SN and of the galaxy have been converted into energy units and divided by the transmission spectrum. Note that a galaxy spectrum has been provided separately to SUPERFIT, and together with the template, it is used to simultaneously fit the observed SN spectrum. The SUPERFIT best match is obtained for the spectrum template used in HSIM sim- ulations and the redshift $z$ is estimated to be $3.000 \pm 0.005$. Fig. 9 displays the spectrum that corresponds to the best fit (black) to the observed spectrum (in blue) at maximum light. The true (noiseless) galaxy spectrum has been subtracted from the observed spectrum and the appropriate fraction from the SUPERFIT best fit. All the first 15 best-fitting templates are SNe Ia, with the top hit being (unsurprisingly) the very spectrum used in the simulation. At maximum light, the spectrum is clearly identified as an SN Ia spectrum. Table 6 gathers all the best match spectrum templates at different ratios, i.e. at different ages of the $\mathrm{SN}$ in this study. Whatever the epoch of observation past maximum light, the best match (and at least the best 15 matches) is obtained for the SN Ia template. As previously mentioned, the same spectrum at maximum light has been used for all the simulations, because the evolution of the spectrum as the SN fades has been ignored in this study. Thus, it is no surprise that the best-fitting spectra from SUPERFIT are always at or near maximum light.

\section{CONCLUSIONS}

We have carried out simulations of SN Ia spectra at $z=3$, using the HSIM simulator, as would be observed in $3 \mathrm{~h}$ using the HARMONI integral field spectrograph being built for the E-ELT. At these redshifts, the rest-frame $B$ - and $V$-band light from the $\mathrm{SN}$ is redshifted into the $H$ and $K$ bands, enabling sensitive observations of well-understood spectral features. The goal of the simulations 
Table 6. List of the best match at different times past maximum light. On the left-hand side, the ratio column represents the ratio $r$ between the SN and galaxy brightness, and the epoch of the simulated $\mathrm{SNe}$ has been estimated from the re-scaled SNe light curve of Perlmutter (2003). The right-hand side corresponds to the best-fitting templates for each ratios, the $S N$ template is given as well as the $S N$ type and the epoch of the best-fitting template in days ( 0 corresponds to the maximum light, -1 corresponds to one day before the maximum light, and -7 corresponds to seven days before the maximum light on the light curve of existing templates).

\begin{tabular}{lcccc}
\hline $\begin{array}{l}\text { Observed SN } \\
\text { Ratio }\end{array}$ & $\begin{array}{c}\text { Epoch } \\
(\mathrm{d})\end{array}$ & $\begin{array}{c}\text { Best-fitting SN } \\
\text { SN }\end{array}$ & SN type & $\begin{array}{c}\text { Epoch } \\
(\mathrm{d})\end{array}$ \\
\hline 0.24 & 0 & $1981 \mathrm{~B}$ & Ia & 0 \\
0.075 & $12.5(1+z)$ & $1981 \mathrm{~B}$ & Ia & 0 \\
0.050 & $18(1+z)$ & $1992 \mathrm{~A}$ & Ia & -1 \\
0.035 & $20(1+z)$ & $1981 \mathrm{~B}$ & Ia & 0 \\
0.024 & $22(1+z)$ & $1989 \mathrm{~B}$ & Ia & -7 \\
\hline
\end{tabular}

was to determine whether (and for how long past maximum light) HARMONI could be used to reliably recover the SN spectral type and redshift.

We find (using two different techniques) that HARMONI spectroscopic observations can reliably recover SN Ia classification for up to $80 \mathrm{~d}$ past maximum light in the observed frame $(20 \mathrm{~d}$ in rest frame) at $z \sim 3$, corresponding to a luminosity ratio (SN/galaxy) of 0.024. The technique employing the SUPERFIT code also recovered the correct redshift of the $\mathrm{SN}$, although spectra of the host galaxy (obtained simultaneously) could also be used to deduce the redshift with high accuracy.

These simulations also imply that if the SN can be observed close to maximum light, spectroscopic characterization/confirmation of SN type could be performed out to redshifts of 7 or 8 ! A detailed study of the ability to robustly detect the SN type as a function of distance from the nucleus and host galaxy redshift is beyond the scope of this work.

The spectral diagnostic relies on broad features in the SN spectrum (e.g. Si II), so substantial spectral smoothing could be used to enhance $\mathrm{S} / \mathrm{N}$ to robustly recover the $\mathrm{SN}$ type. Although we did not simulate the evolution of the observed SN spectrum as it fades from maximum light, we do not expect that the evolution will impact on the result, as long as sufficient features are present in the spectrum. The simulations clearly show that HARMONI will provide spectra at $z \sim 3$ and beyond that are of comparable (or better) quality to the best spectra we can currently obtain at $z \sim 1$.

Our simulations purposely set the SN brightness independent of SN type, although we know that, in fact, SNe Ia are the brightest. In practice, knowing the galaxy redshift, it will be possible to use the spectrophotometry to further constrain/confirm the type classification. By robustly classifying $\mathrm{SN}$ out to $z \sim 3$ and beyond, HARMONI has the potential to substantially improve the use of $\mathrm{SNe}$ Ia as standard candles to measure the $w(z)$ to much larger redshifts. We have shown that the required observing time is reasonable, and that the spectroscopic follow-up observations can be carried out within a month or two.

Typical galaxy sizes at $z \sim 3$ are small, implying that it will be difficult to spatially separate the SN from the galaxy nucleus for many events. However, we observe that the presence of an $\mathrm{SN}$ at or near maximum light significantly increases the total galaxy brightness. Thus, it should be possible to identify SN candidates at high redshift from ground-based surveys based on accurate photometry alone. Even so, finding SN events at high redshift will be difficult.
The enhanced spatial resolution of space telescopes means that it is within the capabilities of space-based missions such as Euclid or JWST, and E-ELT/HARMONI can provide the complimentarity required to make fundamental measurements of the parameters governing our Universe.

\section{ACKNOWLEDGEMENTS}

The authors would like to thank the members of the HARMONI science team for help with this work. SB, NT, SZ, RCWH and MT acknowledge support from the Science and Technology Facilities Council (grants ST/N002717/1 and ST/M007650/1), as part of the UK E-ELT Programme at the University of Oxford.

\section{REFERENCES}

Anderson L. et al., 2012, MNRAS, 427, 3435

Astier P. et al., 2006, A\&A, 447, 31

Astier P. et al., 2014, A\&A, 572, A80

Bailey S. et al., 2009, A\&A, 500, L17

Bazin G. et al., 2011, A\&A, 534, A43

Benetti S. et al., 2004, MNRAS, 348, 261

Bernstein J. P. et al., 2012, ApJ, 753, 152

Betoule M. et al., 2014, A\&A, 568, A22

Beutler F. et al., 2011, MNRAS, 416, 3017

Blondin S., Mandel K. S., Kirshner R. P., 2011, A\&A, 526, A81

Branch D., Lacy C. H., McCall M. L., Sutherland P. G., Uomoto A., Wheeler J. C., Wills B. J., 1983, ApJ, 270, 123

Bronder T. J. et al., 2008, A\&A, 477, 717

Campbell H. et al., 2013, ApJ, 763, 88

Conley A. et al., 2011, ApJS, 192, 1

Davies R. et al., 2016, Proc. SPIE Conf. Ser. Vol. 9908, MICADO: First Light Imager for the E-ELT. SPIE, Bellingham, p. $99081 \mathrm{Z}$

Deffayet C., Dvali G., Gabadadze G., 2002, Phys. Rev. D, 65, 044023

Dvali G., Gabadadze G., Porrati M., 2000, Phys. Lett. B, 485, 208

Filippenko A. V., 1997, ARA\&A, 35, 309

Filippenko A. V. et al., 1995, ApJ, 450, L11

Folatelli G. et al., 2013, ApJ, 773, 53

Foley R. J., Kasen D., 2011, ApJ, 729, 55

Goullioud R. et al., 2012, in Clampin M. C., Fazio G. G., MacEwen H. A., Oschmann J. M., eds, Space Telescopes and Instrumentation 2012: Optical, Infrared, and Millimeter Wave. SPIE, Amsterdam, p. 84421 U

Greenhouse M. A., 2016, in MacEwen H. A., Fazio G. G., Lystrup M., Batalha N., Siegler N., Tong E. C., eds, Space Telescopes and Instrumentation 2016: Optical, Infrared, and Millimeter Wave. SPIE, Edinburgh, p. 990406

Gressler W. J., 2016, in Hall H. J., Gilmozzi R., Marshall H. K., eds, Groundbased and Airborne Telescopes VI. Proc. SPIE, Vol. 9906, p. 99060J

Hamuy M. et al., 2002, AJ, 124, 417

Hogg D., Baldry I., Blanton M., Eisenstein D., 2002, preprint (arXiv: astro-ph/0210394)

Hook I. M., 2013, Phil. Trans. R. Soc. A, 371, 20120282

Hook I. M. et al., 2005, AJ, 130, 2788

Howell D. A. et al. 2005, AJ, 634, 1190

Huterer D., Turner M. S., 1999, Phys. Rev. D, 60, 081301

Ilbert O. et al., 2013, A\&A, 556, A55

Kendrew S. et al., 2016, MNRAS, 458, 2405

Kimm T., Devriendt J., Slyz A., Pichon C., Kassin S. A., Dubois Y., 2011, preprint (arXiv:1106.0538)

Larson D. et al., 2011, ApJS, 192, 16

Laureijs R. et al., 2011, preprint (arXiv:1110.3193)

LSST Science Collaboration et al., 2009, preprint (arXiv:0912.0201)

Maguire K. et al., 2012, MNRAS, 426, 2359

McCarthy P. J. et al., 2016, in Hall H. J., Gilmozzi R., Marshall H. K., eds, Ground-based and Airborne Telescopes VI. Proc. SPIE, Vol. 9906, p. 990612

Menzies J. W. et al., 1987, MNRAS, 227, 39p 
Milne P. A., Foley R. J., Brown P. J., Narayan G., 2015, ApJ, 803, 20

Nordin J. et al., 2011, A\&A, 526, A119

Padmanabhan N., Xu X., Eisenstein D. J., Scalzo R., Cuesta A. J., Mehta K.

T., Kazin E., 2012, MNRAS, 427, 2132

Pan Y.-C., Sullivan M., Maguire K., Gal-Yam A., Hook I. M., Howell D. A., Nugent P. E., Mazzali P. A., 2015, MNRAS, 446, 354

Perlmutter S., 2003, Physics Today. Am. Inst. Phys., New York, p. 53

Perlmutter S. et al., 1999, ApJ, 517, 565

Planck Collaboration I, 2014, A\&A, 571, A1

Powell L. C., Slyz A., Devriendt J., 2011, MNRAS, 414, 3671

Racca G. D. et al., 2016, Proc. SPIE Conf. Ser. Vol. 9904, Space Telescopes and Instrumentation 2016: Optical, Infrared, and Millimeter Wave. SPIE, Bellingham, p. 990400

Reid B. A. et al., 2010, MNRAS, 404, 60

Richardson D., Jenkins III R. L., Wright J., Maddox L., 2014, AJ, 147, 118

Riess A. G. et al., 1998, AJ, 116, 1009

Riess A. G. et al., 2007, ApJ, 659, 98
Riess A. G. et al., 2009, ApJ, 699, 539

Riess A. G. et al., 2011, ApJ, 730, 119

Rodney S. A. et al., 2015, ApJ, 811, 70

Rubin D. et al., 2009, ApJ, 695, 391

Sanders G. H., 2013, JA\&A, 34, 81

Sullivan M. et al., 2011, ApJ, 737, 102

Tamai R., Cirasuolo M., González J. C., Koehler B., Tuti M., 2016, in Hall H. J., Gilmozzi R., Marshall H. K., eds, Ground-Based and Airborne Telescopes VI. Proc. SPIE, Vol. 9906, p. 99060W

Teyssier R., 2002, A\&A, 385, 337

Thatte N. A. et al., 2014, Proc. SPIE Conf. Ser. Vol. 9147, Ground-based and Air-borne Instrumentation for Astronomy V. SPIE, Bellingham, p. 914725

Walker E. S. et al., 2011, MNRAS, 410, 1262

Wright E. L., 2006, PASP, 118, 1711

Zieleniewski S., Thatte N., Kendrew S., Houghton R. C. W., Swinbank A. M., Tecza M., Clarke F., Fusco T., 2015, MNRAS, 453, 3754

\section{APPENDIX A: SIMULATED IMAGES OF THE SUPERNOVA AND HOST GALAXY AT DIFFERENT EPOCHS}

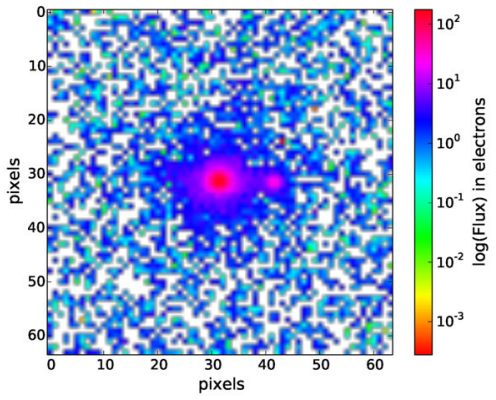

(a) $\mathrm{r}=0.075$

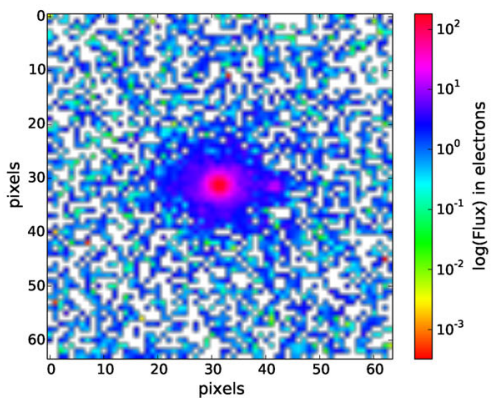

(c) $\mathrm{r}=0.035$

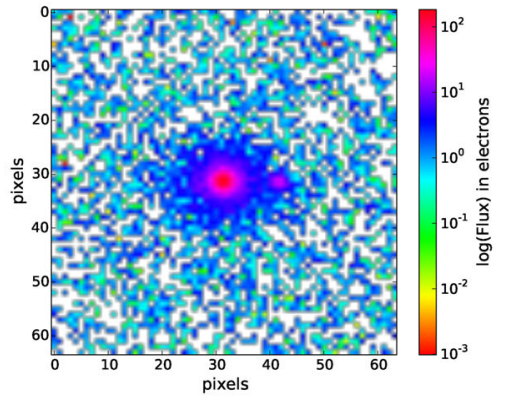

(b) $\mathrm{r}=0.050$

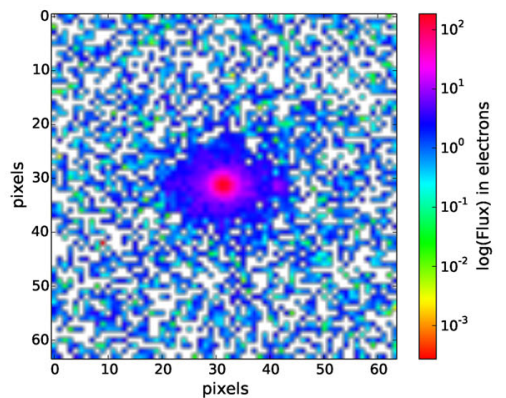

(d) $\mathrm{r}=0.024$

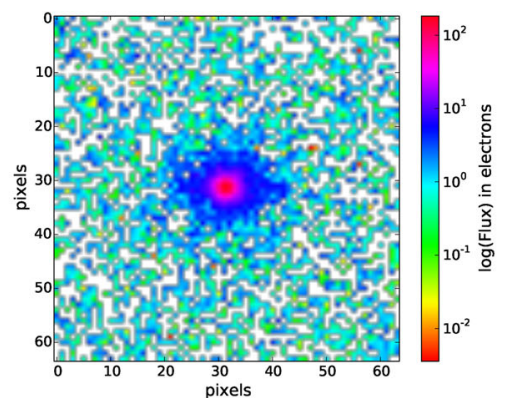

(e) $r=0.010$

Figure A1. View of the observed data cube with the SN within the host galaxy at different times past maximum light (integrated SN/galaxy luminosity ratio $r=0.075,0.050,0.035,0.024$, and 0.01 ). The images are obtained by calculating the median for each spaxel of the sky-subtracted data cube. The SN Ia has been simulated at 0.2 arcsec to the right of the centre of the galaxy, namely 10 spaxels in these simulations. 